Nervenarzt 2021 · 92:556-561

https://doi.org/10.1007/s00115-021-01068-2

Angenommen: 2. Januar 2021

Online publiziert: 11. Februar 2021

(c) Springer Medizin Verlag $\mathrm{GmbH}$, ein Teil von Springer Nature 2021

\author{
Shuyan Liu' · Andreas Heinz ${ }^{1}$ Matthias N. Haucke ${ }^{1,2}$ · Stephan Heinzel ${ }^{2}$ \\ ${ }^{1}$ Klinik für Psychiatrie und Psychotherapie, Charité-Universitätsmedizin Berlin (Campus Charité Mitte), \\ Berlin, Deutschland \\ ${ }^{2}$ Fachbereich Erziehungswissenschaft und Psychologie, Klinische Psychologie und Psychotherapie, Freie \\ Universität Berlin, Berlin, Deutschland
}

\title{
Globale Auswirkungen der COVID-19-Pandemie auf die Versorgung von Menschen mit psychischen Erkrankungen
}

Da die weltweiten COVID-19-Fälle dramatisch zunahmen, initiierten mehrere Länder zwischen Ende März und Mitte Mai 2020 landesweite Erhebungen [19]. Sie verwendeten dasselbe CPDIDesign, das zuvor in China verwendet worden war [23], um die psychische Gesundheit der Allgemeinbevölkerung während dieser anfänglichen Periode hoher Übertragungen von COVID$19 \mathrm{zu}$ beurteilen [19]. Die Ergebnisse zeigen ein weltweit stark variierendes Ausmaß psychischer Belastung, wobei die Prävalenz von niedrig $(6 \%, N=1035$ in Italien und $11,5 \%, N=410$ in $\mathrm{Ne}$ pal) über moderat $(24,1 \%, N=1007$ in Deutschland) bis hoch $(61,1 \%, N=1058$ im Iran und 70,8\%, N=638 in Brasilien) reicht. Verglichen damit berichteten $34,4 \%(N=52.730)$ der Befragten in China psychische Belastungen [18, 19, 23]. Diese Studien unterscheiden sich also erheblich im berichteten Ausmaß psychischer Belastungen. Trotz ungesicherter Repräsentativität weisen sie deutlich darauf hin, dass der Ausbau des Gesundheitssystems, der soziokulturelle und politische Kontext und/oder die eingeleiteten gesundheitspolitischen Maßnahmen Einfluss auf psychische Belastungen während der Pandemie haben [18, 19, 23].

Eine systematische Übersichtsarbeit von Xiong et al. [35] zeigte, dass die Allgemeinbevölkerung in China, Spanien, Italien, Iran, USA, Türkei, Nepal und Dänemark von Beginn der Pandemie bis zum 17.05.2020 eine hohe Stressbelastung und relativ hohe Raten an Angstsymptomen (6,33-50,9\%), depressiven Stimmungszuständen (14,6-48,3\%) und stressbedingten Belastungen (7-53,8\%) berichtete [35]. Anders als in den oben genannten Studien, die alle denselben Fragebogen nutzen, kann die große Heterogenität dieser letztgenannten Ergebnisse auch durch unterschiedliche Bewertungsinstrumente in den verschiedenen Kontexten bedingt sein. Risikofaktoren im Zusammenhang mit psychischer Belastung waren weibliches Geschlecht, jüngeres Lebensalter ( $\leq 40$ Jahre), das Vorliegen chronischer/psychiatrischer Erkrankungen, Arbeitslosigkeit, Studentenstatus und eine häufige Exposition gegenüber Informationen zu COVID19 in sozialen Medien und Nachrichten [35].

Diese Ergebnisse deuten darauf hin, dass es notwendig ist, den psychischen Gesundheitszustand im Verlauf der Pandemie in den einzelnen Ländern wiederholt zu untersuchen und Prädiktoren für die psychische Gesundheit und Gefährdung zu identifizieren. So erhöht Arbeitslosigkeit das Risiko einer Beeinträchtigung der psychischen Gesundheit, insbesondere während einer Rezession und bei begrenzten Beschäftigungsmöglichkeiten [1]. Ein wirtschaftlicher Abschwung kann Angst vor Arbeitsplatzverlust auslösen und das Risiko der sozialen Ausgrenzung erhöhen, was die psychi- 
Hier steht eine Anzeige.

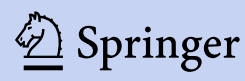


sche Gesundheit deutlich beeinträchtigen kann $[6,7]$.

\section{) Soziale Isolation ist ein wesentlicher Risikofaktor für die Manifestation psychischer Erkrankungen}

Darüber hinaus haben durch die Pandemie und die damit verbundenen Isolationsmaßnahmen bewirkte Einsamkeit und das Ausmaß der selbst wahrgenommenen sozialen Isolation einen starken Einfluss auf das psychische Wohlbefinden [13]. Einerseits spielen soziale Distanzierung, Isolation, Quarantäne und Kontaktbeschränkungen eine Schlüsselrolle bei der Eindämmung der COVID19-Pandemie [32]. Andererseits sind soziale Isolation und eingeschränkte soziale Teilhabe ein wesentlicher Risikofaktor für die Manifestation unterschiedlicher psychischer Erkrankungen inklusive potenziell schwer und chronisch verlaufender Krankheitsbilder wie etwa schizophrener Psychosen, affektiver Erkrankungen und Suchterkrankungen [7, 8]. Eine Metaanalyse von Henssler et al. [9] zeigte anhand der Quarantänemaßnahmen bei früheren Epidemien ein über doppelt so hohes Risiko, an Depressionen oder stressbezogenen Störungen zu erkranken, und auch Angststörungen traten etwa doppelt so häufig auf. Neurobiologisch ist bekannt, dass Stressexposition zum Auftreten von negativen Stimmungszuständen und erhöhter Aggressivität beitragen kann [8]. Soziale Unterstützung gilt demgegenüber als Schutzfaktor $[2,7]$.

Psychische Belastungen sind dann besonders ausgeprägt, wenn zusätzlich zur sozialen Isolation auch eine wirtschaftliche Notlage auftritt. Dies wurde im Rahmen der Austeritätspolitik und ihren Folgen während der Finanzkrise in Griechenland gut belegt [6]. In diesem Kontext wurde in der Rezession (2009-2015) in Griechenland ein Anstieg der Suizidraten um 33\% beobachtet [6]. Dazu passend zeigte sich in einer Umfrage in Deutschland, dass das Ausmaß der erlebten psychischen Belastungen nicht mit der Zahl infizierter Personen, sondern mit der Höhe der Arbeitslosigkeit korrelierte [19]. Hinzu kommen Hinweise, dass ein erhöhter Drogen- und insbesondere Alkoholkonsum während sozialer Isolation zu einer erhöhten Zahl häuslicher Gewalttaten führen kann [8].

\section{Psychische Belastung und Beanspruchung von Patientinnen und Patienten}

Während der COVID-19-Pandemie kommt es weltweit zu einem Anstieg depressiver Symptome und Störungen, die laut aktuellen Studien aus den USA während der Pandemie etwa dreimal häufiger auftraten als zuvor [5, 29]. Zudem können sich bereits bestehende Erkrankungen verschlechtern. So stellte die COVID-19-Pandemie auch Patientinnen und Patienten mit Essstörungen wie Anorexia nervosa, Bulimia nervosa und Binge-Eating-Störung vor große Herausforderungen [26].

Diese Berichte werden auch durch systematische Reviews und Metaanalysen bestätigt, wonach soziale Isolation nicht nur zur einer Zunahme von Angststörungen und affektiven Erkrankungen sowie stressbedingten psychischen Problemen führen kann, sondern auch bestehende Erkrankungen verschlechtert $[9,11]$. Bei Personen, die sich mit SARS-CoV-2 infiziert haben, kommen direkte Wirkungen der Infektion hinzu. So können Symptome eines hirnorganischen Psychosyndroms im Sinne eines Deliriums auftreten, im weiteren Verlauf auch affektive Störungen, Angststörungen, Fatigue und andere psychische Beschwerden [25, 28].

\section{)> Der Zugang zu am- bulanten und stationären Hilfsangeboten ist durch die Isolationsmaßnahmen erschwert}

Ein möglicher Grund dafür, dass bereits bestehende Erkrankungen in ihrem Verlauf durch soziale Isolations- und Quarantänemaßnahmen negativ beeinflusst werden [9], ist neben den Auswirkungen der sozialen Isolation selbst die oft stark eingeschränkte Zugänglichkeit von ambulanten und stationären psy- chiatrischen und psychotherapeutischen Hilfsangeboten. So wurden persönliche Kontakte in vielen Ambulanzen ersetzt durch Kontakte über Videokonferenzen oder Telefonate, was aber insbesondere für Menschen mit sozialen Einschränkungen die Zugänglichkeit reduzieren kann. Nach aktuellen Zahlen des Zentralinstituts für die kassenärztliche Versorgung in Deutschland ging die Inanspruchnahme von Einzel- und Gruppenpsychotherapie und der Substitutionsbehandlung im ersten Halbjahr 2020 deutlich zurück, während kassenärztliche Videosprechstunden von knapp 600 im ersten Halbjahr 2019 auf über 1.000.000 im Vergleichszeitraum 2020 anstiegen [36].

\section{International empfohlene Maßnahmen zur Minderung der Folgen}

Die Weltgesundheitsorganisation (WHO; [34]), der Ständige interinstitutionelle Ausschuss der Vereinten Nationen [27] sowie die Internationale Föderation der Rotkreuz- und Rothalbmond-Gesellschaften [14] fassen internationale Empfehlungen zum Umgang mit psychischer Belastung und Beanspruchung während der COVID-19-Pandemie zusammen, um Handlungsoptionen für praktizierende Ärztinnen und Ärzte und andere Gesundheitsfachkräfte zu vermitteln.

Die WHO $[33,34]$ gibt unter anderem folgende Empfehlungen für individuelle Bewältigungsstrategien bezüglich COVID-19-bezogener psychischer Belastungen (hier zusammenfassend dargestellt):

- Kontakte zu Freunden und Familie

- Förderung eines gesunden Lebensstils, z. B.

- körperliche Aktivität,

- gesunde Ernährung und

- soziale Kontakte, gegebenenfalls über Telefon/elektronische Medien

- Vermeiden von Tabak, Alkohol oder anderen Drogen zur Emotionsregulation

- Im Bedarfsfall professionelle Unterstützung bei Gesundheitsfachkräften suchen

- Orientierung an Fakten bezüglich des persönlichen Risikos und Mög- 
lichkeiten, sich zu schützen (in Deutschland z. B. über das Robert Koch-Institut)

- Reduktion der Zeit mit Konsum beunruhigender Medienberichte

$\mathrm{Zu}$ spezifischen Hinweisen für ältere Menschen und Kinder sei an dieser Stelle auf die Übersichten von Petzold et al. [20] und Liu et al. [17] verwiesen. Eine aktuelle Umfrage in Deutschland [18] zeigte, dass körperliche Aktivität (z.B. Joggen), kognitive Aktivitäten (z. B. Lesen) sowie virtuelle soziale Kontakte von den Befragten als hilfreiche Strategien angesehen wurden.

\section{) Auf politischer und sozialer Ebene gilt es, den Zusammenhalt der Gesellschaft zu stärken}

Um die unmittelbaren und langfristigen psychischen Folgen von COVID-19 zu bewältigen, ist es auf politischer und sozialer Ebene wichtig, den Zusammenhalt der Gesellschaft zu stärken [22] und die Gesundheitsversorgung sicherzustellen. Hier wird vom IASC [27] unter anderem empfohlen, den Zugang zu Präventionsund Therapieangeboten insbesondere für vulnerable Gruppen zu verbessern, allen Bevölkerungsgruppen akkurate und aktuelle Informationen über COVID-19 zur Verfügung zu stellen sowie Mitarbeiterinnen und Mitarbeiter im Gesundheitssystem darin zu schulen, psychische Störungen und Krisen zu identifizieren und adäquat zu versorgen.

Qi et al. [22] stellten fest, dass während des COVID-19-Ausbruchs ein geringeres $\mathrm{Maß}$ an sozialer Unterstützung mit einer höheren Prävalenz psychischer Gesundheitsprobleme bei Jugendlichen verbunden war. In China waren Wohneinheiten mit dem Namen Shèqū eine wichtige Komponente im Kampf gegen SARSCoV-2: Gemeinden im Shèqū-System lieferten Lebensmittel aus und sammelten Hausabfälle für diejenigen, die sich selbst isolieren mussten. Weltweit spielen niedrigschwellige kommunale psychosoziale Hilfsangebote insbesondere für Menschen, die sich in Quarantäne befinden, eine Schlüsselrolle im Kampf gegen die psychischen Folgen der COVID-19-

Nervenarzt 2021 · 92:556-561 https://doi.org/10.1007/s00115-021-01068-2

(c) Springer Medizin Verlag GmbH, ein Teil von Springer Nature 2021

\section{S. Liu · A. Heinz $\cdot$ M. N. Haucke $\cdot$ S. Heinzel}

\section{Globale Auswirkungen der COVID-19-Pandemie auf die Versorgung von Menschen mit psychischen Erkrankungen}

\section{Zusammenfassung}

Mit zunehmend umfangreicheren Daten zu den psychischen Folgen der Coronavirusdisease-2019(COVID-19)-Pandemie wird deutlich, dass die psychische Belastung der Bevölkerung durch die Pandemie in vielen Regionen der Welt stark zugenommen hat. Neben der Angst vor einer Infektion mit dem "severe acute respiratory syndrome coronavirus 2" (SARS-CoV-2) führen vielerorts auch die Maßnahmen zur Eindämmung der Pandemie zu psychischen Belastungen. So wurde gezeigt, dass soziale Distanzierung und Kontaktbeschränkungen Risikofaktoren für die Entstehung oder Verschlechterung psychischer Störungen sind. Gleichzeitig hat sich die Versorgung von Menschen mit psychischen Störungen während der Pandemie oft verschlechtert, sodass ein erheblicher Bedarf an niedrigschwelligen
Hilfsangeboten und Bewältigungsstrategien besteht. Aktuelle Studien konnten zeigen, dass bestimmte Coping-Strategien wie virtuelle soziale Kontakte und körperliche Aktivität den negativen psychischen Folgen der Pandemie entgegenwirken können. Um jedoch mittelfristig die Unterversorgung psychisch belasteter und erkrankter Personen zu reduzieren, bedarf es einer gezielten Ausweitung des Behandlungsangebots, insbesondere der Onlinepsychotherapie, sowie der Förderung von Maßnahmen, die Armut oder soziale Isolation verhindern.

\section{Schlüsselwörter}

Psychische Gesundheit · Soziale Isolation . Virtuelle soziale Kontakte und körperliche Aktivität · Präventionsrichtlinie · Coping Strategien

\section{The global impact of the COVID-19 pandemic on the care provision for people with mental health problems}

\section{Abstract}

There are increasingly comprehensive data on recognition of the psychological impact of the coronavirus disease 2019 (COVID 19) pandemic on global populations. In most regions of the world, the economic and psychological burden on the general population and persons with mental disorders has risen sharply over the course of the pandemic. Beyond anxieties regarding severe acute respiratory syndromes due to coronavirus type 2 (SARS-CoV-2) infections, in many countries the measures for containing the pandemic also led to psychological burdens. It was shown that social distancing and contact restrictions were risk factors for the development or deterioration of mental health problems on a global scale. Simultaneously, the COVID-19 pandemic has disrupted or reduced mental health services in many countries. Thus, there is a substantial need for low threshold offers of assistance and for coping strategies. Current studies could show that certain coping strategies, such as virtual social contacts and physical activity, appear to be useful in counteracting the negative psychological sequelae of the pandemic; however, in order to improve care provision for people with psychological burden and mental illness in the medium term, it is necessary to offer targeted treatment, such as online psychotherapy in particular. Collaborative research tackles poverty, social isolation and loneliness.

\section{Keywords}

Mental health - Social isolation - Virtual social networking and physical activity . Prevention policy. Coping strategies
Pandemie, wozu auch die Einrichtung spezifischer Telefonhotlines und Onlinehilfsangebote für psychische Gesundheit bzw. psychotherapeutische Beratung gehört [31]. Für den deutschsprachigen Raum verweisen wir auf eine Übersicht der Bundespsychotherapeutenkammer (BPtK) $\mathrm{zu}$ telefonischen Hilfsangebo- ten [3] sowie auf eine Übersicht der Deutschen Gesellschaft für Psychiatrie und Psychotherapie, Psychosomatik und Nervenheilkunde (DGPPN) zu Onlineberatungs- und Therapieangeboten bzw. E-Mental-Health-Anwendungen [4].

Die Auswirkungen dieser Pandemie können soziale Unterschiede verstärken, 
die in mehreren Studien mit dem Auftreten psychischer Erkrankungen korrelierten $[7,21,24]$. Im internationalen Maßstab sollten künftige Studien die Auswirkungen sozialer Ungleichheit und politischer Teilhabemöglichkeiten auf die psychische Gesundheit vergleichen und den Einfluss unterschiedlicher Versorgungssysteme beschreiben.

\section{Fazit für die Praxis}

Aus den zitierten Studien und Berichten ergeben sich die folgenden Handlungsempfehlungen:

- Eine bessere Versorgung der Bevölkerung mit therapeutischen Angeboten, gerade im Bereich der Angststörungen, affektiven Erkrankungen sowie stressbedingten psychischen Probleme, sollte Videosprechstunden und telefonische Angebote beinhalten.

- Eine Erhöhung therapeutischer Onlineangebote sollte zudem besonders auf diejenigen Personen ausgerichtet werden, die stark unter den Folgen der Pandemie leiden: Arbeitssuchende, junge Menschen, Frauen, Alleinerziehende und sozial isolierte Menschen.

- Ein besseres Informations- und Beratungsangebot wird für Frauen benötigt, die häusliche Gewalt erfahren.

- Informations- und Beratungsangebote gegen soziale Isolation sollten auch in leichter Sprache vorliegen. Dazu gehören auch Empfehlungen von Onlinesportangeboten oder sozialen Onlineveranstaltungen.

- Wichtig zur Vermeidung ausgeprägter negativer Folgen sozialer Isolation sind Treffen mit einzelnen Personen unter Einhaltung der geltenden Infektionsschutzmaßnahmen und Kontaktbeschränkungsregelungen. Treffen sollten in geeigneter Form auch für ältere, in Heimen lebende Personen ermöglicht werden.

\section{Korrespondenzadresse}

\section{Shuyan Liu}

Klinik für Psychiatrie und Psychotherapie, Charité-Universitätsmedizin Berlin (Campus Charité Mitte)

Charitéplatz 1, 10117 Berlin, Deutschland siyan908@hotmail.com

Danksagung. Gefördert im Rahmen der Exzellenzstrategie von Bund und Ländern durch die Berlin University Alliance (Projekt an SL und SH). Mit Unterstützung der Deutschen Forschungsgemeinschaft (Projekt SFB/TRR 265 an AH). Wir danken dem gesamten Team von Siuvo Inc, dem Shanghai Mental Health Center, dem World Health Summit und der Deutschen Gesellschaft für Psychiatrie und Psychotherapie, Psychosomatik und Nervenheilkunde e. V. (DGPPN). Die Autoren danken Frau Emily Giulia Schütte, Frau Virginia Eckleben und Frau Luisa Wegner für die Hilfe beim Erstellen des Manuskripts.

\section{Einhaltung ethischer Richtlinien}

Interessenkonflikt. S. Liu, A. Heinz, M.N. Haucke und S. Heinzel geben an, dass kein Interessenkonflikt besteht.

Für diesen Beitrag wurden von den Autoren keine Studien an Menschen oder Tieren durchgeführt. Für die aufgeführten Studien gelten die jeweils dort angegebenen ethischen Richtlinien.

\section{Literatur}

1. Bartelink VH, Zay YaK, Guldbrandsson Ket al (2020) Unemployment among young people and mental health: a systematic review. Scand J Public Health 48:544-558

2. Brooks SK, Webster RK, Smith LE et al (2020) The psychological impact of quarantine and how to reduce it: rapid review of the evidence. Lancet 395:912-920

3. Bundespsychotherapeutenkammer. https:// www.bptk.de/wp-content/uploads/2020/ 04/BPtK-Wegweiser-für-psychisch-krankeMenschen-in-der-Coronakrise.pdf. Zugegriffen: April 2020

4. Deutsche Gesellschaft für Psychiatrie und Psychotherapie, Psychosomatik und Nervenheilkunde. https://dgppn.de/schwerpunkte/e-mentalhealth/corona.html.Zugegriffen:05.02.2021

5. Ettman CK, Abdalla SM, Cohen GH et al (2020) Prevalence of depression symptoms in US adults before and during the COVID-19 oandemic. JAMA Netw Open 3:e2019686

6. Fountoulakis KN (2020) Suicides in Greece before and during the period of austerity by sex and age group: relationship to unemployment and economic variables. J Affect Disord 260:174-182

7. Heinz A, Zhao X, Liu S (2020) Implications of the association of social exclusion with mental health. JAMA Psychiatry 2:113-114

8. Heinz AJ, Beck A, Meyer-Lindenberg $A$ et al (2011) Cognitive and neurobiological mechanisms of alcohol-related aggression. Nat Rev Neurosci 12:400-413
9. Henssler J, Stock F, van Bohemen J et al (2020) Mental health effects of infection containment strategies: quarantine and isolation - a systematic review and meta-analysis. Eur Arch Psychiatry Clin Neurosci. https://doi.org/10.1007/s00406-02001196-x

10. Holmes EA, O'Connor RC, Perry VH et al (2020) Multidisciplinary research priorities for the COVID19 pandemic: a call for action for mental health science. Lancet Psychiatry 7:547-560

11. Hossain MM, Sultana A, Purohit N (2020) Mental health outcomes of quarantine and isolation for infection prevention: a systematic umbrella review of the global evidence. Epidemiol Health 42:e2020038. https://doi.org/10.4178/ epih.e2020038

12. Huang Y, Zhao N (2020) Generalized anxiety disorder, depressive symptoms and sleep quality during COVID-19 outbreak in China: a web-based cross-sectional survey. Psychiatry Res 288:112954

13. Hwang T-J, Rabheru K, Peisah C et al (2020) Loneliness and social isolation during the COVID19 pandemic. Int Psychogeriatr 32:1217-1220

14. Internationale Föderation der Rotkreuz- und Rothalbmond-Gesellschaften. https://pscentre. org/wp-content/uploads/2020/02/MHPSS-innCoV-2020_ENG-1.pdf.Zugegriffen: Februar 2020

15. Johns Hopkins University Coronavirus Resource Center. https://coronavirus.jhu.edu/. Zugegriffen: 31.10.2020

16. Kaufman KR, PetkovaE, Bhui KS et al (2020) A global needs assessment in times of a global crisis: world psychiatry response to the COVID-19 pandemic. BJPsych open 6:e48

17. Liu JJ, Bao Y, Huang X et al (2020) Mental health considerations for children quarantined because of COVID-19. Lancet Child Adolesc Health 4:347-349

18. Liu S, Heinz A (2020) Cross-cultural validity of psychological distress measurement during the coronavirus pandemic. Pharmacopsychiatry 53:237-238

19. Liu S, Heinzel S, Haucke MN et al (2021) Increased psychological distress, loneliness and unemployment in the spread of COVID-19 over 6 months in Germany. Medicina 57(1):53. https:// doi.org/10.3390/medicina57010053

20. Petzold MB, Ströhle A, Plag J (2020) COVID-19-Pandemie: Psychische Belastungen können reduziert werden. Dtsch Arztebl Int 117:A648-A654

21. Pickett KE, Wilkinson RG (2015) Income inequality and health: a causal review. Soc Sci Med 128:316-326

22. Qi M, Zhou S-J, Guo Z-C et al (2020) The effect of social support on mental health in Chinese adolescents during the outbreak of COVID-19. JAdolesc Health 67:514-518

23. Qiu J, Shen B, Zhao M et al (2020) A nationwide survey of psychological distress among Chinese people in the COVID-19 epidemic: implications and policy recommendations. Gen Psychiatr 33(2):e100213. https://doi.org/10.1136/gpsych2020-100213

24. Rapp MA, Kluge U, Penka S et al (2015) When local poverty is more important than your income: mental health in minorities in inner cities. World Psychiatry 14:249-250

25. Rogers JP, Chesney E, Oliver D et al (2020) Psychiatric and neuropsychiatric presentations associated with severe coronavirus infections: a systematic review and meta-analysis with comparison to the COVID-19 pandemic. Lancet Psychiatry 7(7):611-627. https://doi.org/10.1016/ S2215-0366(20)30203-0 
26. Schlegl S, Maier J, Meule A et al (2020) Eating disorders in times of the COVID-19 pandemic - results from an online survey of patients with anorexia nervosa. Int JEat Disord 53:1791-1800

27. Ständige interinstitutionelle Ausschuss. https://interagencystandingcommittee.org/ iasc-reference-group-mental-health-andpsychosocial-support-emergency-settings/ interim-briefing. Zugegrifen: 17.03.2020

28. Taquet M, Luciano S, Geddes J et al (2021) Bidirectional associations between COVID-19 and psychiatric disorder: retrospective cohort studies of 62,354 COVID-19 cases in the USA. Lancet Psychiatry 8(2):130-140. https://doi.org/10.1016/ S2215-0366(20)30462-4

29. Twenge JM, Joiner TE (2020) US census bureauassessed prevalence of anxiety and depressive symptoms in 2019 and during the 2020 COVID-19 pandemic. Depress Anxiety 37:954-956

30. Wang C, Pan R, Wan X et al (2020) Immediate psychological responses and associated factors during the initial stage of the 2019 coronavirus disease (COVID-19) epidemic among the general population in China. IJERPH 17:1729

31. Wang Y, Zhao X, Feng Q et al (2020) Psychological assistance during the coronavirus disease 2019 outbreak in China. J Health Psychol 25(6):733-737. https://doi.org/10.1177/1359105320919177

32. Wilder-Smith A, Freedman D (2020) Isolation, quarantine, social distancing and community containment: pivotal role for old-style public health measures in the novel coronavirus (2019nCoV) outbreak. J Travel Med 27:1-4

33. World Health Organization (2020) Coping with stress during the 2019-nCoV outbreak. https:// www.who.int/docs/default-source/coronaviruse/ coping-with-stress.pdf.Zugegriffen: 05.02.2021

34. World Health Organization (2020) Mental health and psychosocial considerations during the COVID-19 outbreak. https://www.who.int/docs/ default-source/coronaviruse/mental-healthconsiderations.pdf. Zugegriffen: 18.03 .2020

35. Xiong J, Lipsitz O, Nasri F et al (2020) Impact of COVID-19 pandemic on mental health in the general population: a systematic review. J Affect Disord 277:55-64

36. Zentralinstitut für die kassenärztliche Versorgung der Bundesrepublik Deutschland. https://www. zi.de/fileadmin/images/content/Publikationen/ Trendreport 2 Leistungsinanspruchnahme COVID_2020-11-11.pdf.Zugegriffen:11.11.2020

\section{Master of Migraine and Headache Medicine (MMHM)}

Neuer berufsbegleitender akademischer Studiengang an der Universität Kiel

An der Universität Kiel ist zum WS 2021/22

der neue akademische Studiengang Master of Migraine and Headache Medicine (MMHM) geplant. Das Studium ist berufsbegleitend über vier Semester organisiert. Die Vorlesungen finden an Wochenenden und in Präsenzwochen statt. Der Masterstudiengang vermittelt die zeitgemäßen Neuentwicklungen in der Migräne- und Kopfschmerztherapie. Kern ist ein fachübergreifendes Konzept mit theorie- und praxisorientierten Modulen. Der universitäre Studiengang schließt mit dem akademischen Grad Master of Arts (M.A.) Migraine and Headache Medicine (MMHM) ab.

Migräne und Kopfschmerzerkrankungen sind nach Karies die bedeutsamsten Volkskrankheiten. Jedoch erhalten weniger als $20 \%$ der Betroffenen eine leitliniengerechte Prävention oder Akuttherapie. Der Bedarf an zeitgemäßer Versorgung ist hoch. Das Wissen zur Klassifikation, Diagnostik und Therapie hat sich erheblich erweitert. Aktuelle Grundlagenforschung und klinische Studien wurden die Basis für eine zeitgemäße Behandlung. Das individuelle Leid und die große gesellschaftliche Bedeutung erfordern eine Schwerpunktsetzung. Damit steigt der Bedarf an qualifizierten fachübergreifenden Migräne- und Kopfschmerzexpert:innen. Die Schmerzklinik Kiel und die Medizinische Fakultät der Universität Kiel haben deshalb den Masterstudiengang Migraine and Headache Medicine (MMHM) entwickelt. Renommierte und qualifizierte Dozent:innen präsentieren einen fachübergreifenden akademischen Wissenskanon. Studierende können zudem aktiv ihre unterschiedlichen Berufserfahrungen und Fachkenntnisse einbringen. Verschiedene Lehrformen wie Fallseminare, Kliniktage und Gruppendiskussionen bieten dafür umfangreiche Voraussetzungen. Ein zentrales Element sind mannigfaltige praktische Veranstaltungen im ambulanten und klinischen Bereich.

Der Studiengang wird berufsbegleitend organisiert. Die Präsenzphasen finden in den ersten drei Semestern an 15 Wochenenden auf dem Campus statt. In drei Präsenzwochen wird ein Klinikpraktikum an der Schmerzklinik Kiel absolviert. Die Bearbeitung der Masterarbeit erfolgt im 4. Semester. Wäh- rend dieser Zeit sind keine Präsenzzeiten vorgesehen.

Umfassende theoretische Ausbildung und gleichzeitig eine prägnante Praxisorientierung stehen im Mittelpunkt. In drei Semestern werden Grundlagen und Therapie vermittelt. Zentrale Themen sind dabei auch Organisation und Strukturen der Behandlung. Im vierten Semester folgt die fachgebundene Masterarbeit. Es handelt sich um einen universitären Studiengang mit hohem fachlichem Niveau. Themen wie DRGs, multimodale Behandlung, ambulante und stationäre Versorgung finden u.a. eingehend Berücksichtigung.

Zugangsvoraussetzung ist ein Hochschulstudium mit mindestens 240 Leistungspunkten. Möglich sind z.B. Human- oder Zahnmedizin, Psychologie oder ein verwandtes Fach. Ebenfalls möglich sind Gesundheits-,

Pflege-, Natur- und technische Wissenschaften. Zusätzlich muss eine mindestens zweijährige Berufserfahrung im Bereich Medizin oder Psychologie vorliegen.

Das Zentrum für wissenschaftliche Weiterbildung an der Universität organisiert den Studiengang. In vielen Fällen ist es möglich, dass die steuerlich abzugsfähigen Studiengebühren vom Arbeitgeber übernommen werden. Des Weiteren ist die Möglichkeit der finanziellen Förderung durch Stipendien geplant.

Detaillierte Infos finden sich unter: https://www.zww-kiel.de/migraineheadache-medicine

Zentrum für wissenschaftliche Weiterbildung (ZWW)

c/o Universitätsklinikum Schleswig-Holstein, Campus Kiel

Arnold-Heller-Straße 3, Haus U27

24105 Kiel

Telefon: 0431 - 50024462

Telefax: 0431 - 50020268

E-Mail:kopfschmerz@zww.uni-kiel.de

Studiengangsleitung:

Prof. Dr. med. Dipl.-Psych. Hartmut Göbel

E-Mail:hg@schmerzklinik.de 